\title{
P03-132
}

\section{THE EFFECT OF QUETIAPINE ON AUDITORY P300 RESPONSE IN PATIENTS WITH SCHIZOAFFECTIVE DISORDER: PRELIMINARY STUDY}

M. Korostenskaja ${ }^{1,2,3}$, K. Dapsys ${ }^{1}$, A. Siurkute ${ }^{1}$, A. Dudlauskaite ${ }^{1}$, A. Pragaraviciene ${ }^{4}$, V. Maciulis ${ }^{1}$, S. Kähkönen ${ }^{3,5}$

${ }^{1}$ Republican Vilnius Psychiatric Hospital, Vilnius, Lithuania, ${ }^{2}$ Division of Neurology, Cincinnati Children's Hospital Medical Center, Ohio, USA, ${ }^{3}$ BioMag Laboratory, Helsinki University Central Hospital, Helsinki, Finland, ${ }^{4}$ Department of Psychology and Didactics, Faculty of Pedagogy and Psychology, Vilnius, Lithuania, ${ }^{5}$ Cognitive Brain Research Unit, Helsinki University, Helsinki, Finland

Abnormalities in attention, memory and information processing are considered to be the primary deficits in schizophrenia. Eventrelated potential (ERP) P300 could reflect deficits in auditory information processing related to active attention in schizophrenia patients. Atypical antipsychotics tend to ameliorate cognitive deficits, however their effects on neural aspects of cognitive dysfunction have not been consistent.

Aim: To investigate the effects of quetiapine on auditory information processing by using auditory P300.

Methods: We examined 7 patients with schizoaffective disorder, depressive type and 7 age- and sex-matched healthy controls. ERPs were elicited during active auditory "oddball" paradigm. P300 was recorded before and after two weeks of treatment with quetiapine ( $468.7 \pm 143 \mathrm{mg} /$ day).

Results: Baseline P300 latencies were significantly delayed in patients compared with controls. Quetiapine did not change P300 amplitudes. However, it normalized P300 latency. These results suggest that already after two weeks of treatment, quetiapine could have a beneficial effect on the active attention reflected in P300 in patients with schizoaffective disorder. Previous studies with antipsychotic drugs such as olanzapine and risperidone failed to show changes in P300 after this treatment interval.

Conclusion: Quetiapine may be faster than other neuroleptics in ameliorating attentional dysfunction in patients with schizoaffective disorder. However, studies with a larger sample size must be conducted in order to confirm or reject the results of the current study. 